\title{
Association of dental and periodontal status with bisphosphonate-related osteonecrosis of the jaws. A retrospective case controlled study
}

\author{
Marcin Kos
}

Department of Maxillofacial Surgery, Klinikum Minden, Germany

Submitted: 16 March 2012

Accepted: 25 November 2012

Arch Med Sci 2014; 10, 1: 117-123

DOI: $10.5114 /$ aoms.2014.40738

Copyright (C) 2014 Termedia \& Banach

\section{Abstract}

Introduction: To assess the association of oral hygiene, dental caries, and periodontal status with bisphosphonate-related osteonecrosis of the jaws. Material and methods: A retrospective case-control study on 81 patients treated for neoplasms with bone metastases. Twenty-nine patients with bone necrosis and 52 controls treated with bisphosphonates were compared using the Oral Hygiene Index, Decay, Missing, Filled Teeth, Community Periodontal Index of Treatment Needs, and Residual Periodontal Bone. The null hypothesis stated that there was no difference in parameters of oral health between patients with and without bone necrosis. Differences of means of above-mentioned variables were compared between the groups with Student's $t$-test or Mann-Whitney rank sum test and $\chi^{2}$ test. Value of $p \leq 0.05$ was considered significant.

Results: Poorer oral hygiene (OHIs 1.94 vs. $1.32 ; p=0.065$ ), more advanced dental caries (DMFT 26.85 vs. 22.87; $p=0.05$ ), and more advanced periodontal disease $($ CPITN: $=0: 21.05 \%$ vs. $42.51 \% ;=113.16 \%$ vs. $7.29 \% ;=2: 0 \%$ vs. $15.38 \%$; $=3: 65.79 \%$ vs. $28.34 \% ;=4: 0 \%$ vs. $6.48 \%$, Residual periodontal bone $73.1 \%$ vs. $80.51 \%$; $p=0,001$ ) were characteristic of patients with bisphosphonate related jaw necrosis when compared with control group. An advanced dental caries or periodontal disease required surgical intervention which directly contributed to the development of the bone necrosis.

Conclusions: Dental and periodontal disease can lead to bisphosphonate-related osteonecrosis of the jaw. Oncologic patients treated with bisphosphonates should be offered preventive care to reduce dental plaque, calculus, dental caries, and periodontal disease.

Key words: necrosis, jaw, dental status, periodontal status, oral health.

\section{Introduction}

Due to their unique ability to suppress osteoclast activity, bisphosphonates (BPs) are currently one of the most potent recognized inhibitors of bone resorption $[1,2]$. They have been used for several years in the treatment of osteoporosis, Paget's disease, and fibrous dysplasia [3, 4]. They have also become part of chemotherapeutic protocols for the management of bone-invasive tumors [5]. During this time BPs have been considered efficacious drugs with few adverse effects due to their strong affinity for bone tissue and minimal metabolism [2]. However, since 2003 there have been increasing reports of large and therapy-resis-

\author{
Corresponding author: \\ Marcin Kos MD, DDS, PhD \\ Department \\ of Maxillofacial Surgery \\ Klinikum Minden \\ Hans-Nolte-Strasse 1 \\ 32429 Minden, Germany \\ Phone: + 495717903701 \\ Fax: +49 571790293700 \\ E-mail: mkos@poczta.onet.pl
}


tant jaw bone exposures in patients treated with BPs [6-9]. This phenomenon is observed primarily after infusion of potent, nitrogen-containing $\mathrm{BPs}$, and is referred to as bisphosphonate-related osteonecrosis of the jaws (BRONJ). However, this process is incompletely understood on the epidemiologic, pathophysiological, and treatment levels. The reported incidence of BRONJ varies from 0 to $28 \%$ in different settings, being affected by the type of underlying disease, population studied, and applied methodology [10-13]. Questions concerning the predilection for jaw involvement, association with oral surgical procedures, and reasons for colonization of the denuded bone by specific bacterial strains remain unanswered. Furthermore, there is a deficit of studies concerning the influence of local factors, i.e. dental plaque, caries, or periodontitis, on the development of BRONJ. Poor oral health is discussed widely in the BRONJ literature, but research work with high levels of clinical evidence is missing [14]. Meanwhile, oncological patients often present with impaired dental hygiene and are susceptible to oral infections [15-17]. On the other hand, there are reports of a positive influence of BPs on the clinical outcome of periodontal therapy and preservation of periodontal bone mass [18-21]. These controversial data raise the question of whether local factors such as dental caries, oral hygiene, and periodontal status might play a role in the development of BRONJ. To address this question, a comparative analysis of oncologic patients treated with BPs was conducted.

\section{Material and methods}

\section{Study design and study population}

This was a retrospective control case study of 81 patients with multiple myeloma or solid tumors invading the skeleton. All patients received BPs as part of their treatment protocol. In addition to chemotherapy, intravenous infusions of $60 \mathrm{mg}$ or $90 \mathrm{mg}$ pamidronate, $4 \mathrm{mg}$ zoledronate, or $6 \mathrm{mg}$ ibandronate were administered every 3 to 4 weeks. Some of the patients received more than one type of BP. Seventy-two patients were treated in the Department of Oncology and Hematology, Klinikum Minden, Germany, between 1 January 2008 and 31 December 2009; the remainder were referred to the Department of Maxillofacial Surgery, Klinikum Minden, during the same time frame from other oncologic centers for suspicion of BRONJ. Because of awareness of the risk of BRONJ, all oncologic patients on BPs at admission were routinely examined at the Department of Maxillofacial Surgery by the author on the basis of established cooperation between the oncology and maxillofacial surgery departments. Clinical examination including reg- istration of dental plaque, dental calculus, caries, and assessment of periodontal status was completed by dental panoramic views. The BRONJ was diagnosed when BP administration was followed by bone exposure that did not heal within eight weeks of identification in patients with no history of local radiation. The patients without symptoms of BRONJ served as a control group. The study was conducted in full accordance with ethical principles of the Declaration of Helsinki (version 2008).

\section{Registration of explanatory variables}

The following data from clinical charts were collected for each patient: sex, age, underlying diagnosis and period of oncologic disease, type of $\mathrm{BP}$, duration of its administration, and diagnosis of BRONJ when present. The recorded oral status and radiographs were used to calculate the indices as follows: the DMFT index, namely $D$ (decayed), $M$ (missing), and $F$ (filled) teeth, were recorded and the $T$ (teeth) value for each subject was obtained by summing D, M, and F components. The Simplified Oral Hygiene Index (OHI-S) was calculated after the scores for debris and calculus were recorded on the teeth surfaces according to the following range: 0 - no debris/calculus present; 1 - debris/ calculus covering not more than $1 / 3$ of the tooth surface; 2 - debris/calculus covering not more than $2 / 3$ of the tooth surface or the presence of individual flecks of subgingival calculus around the cervical portion of the tooth; 3 -debris/calculus covering more than $2 / 3$ of the exposed tooth surface or a continuous heavy band of subgingival calculus around the cervical portion of the tooth. Examination of at least two of the six possible surfaces selected from four posterior and two anterior teeth was required for an individual score to be calculated. In the posterior portion of the dentition, the first fully erupted tooth distal to the second bicuspid, usually the first molar, was examined. The buccal surfaces of the selected upper molars and the lingual surfaces of the selected lower molars were inspected. In the anterior portion of the mouth, the labial surfaces of the upper right and the lower left central incisors were scored. For each individual, the debris and calculus scores were totaled and divided by the number of surfaces scored, then the average individual debris and calculus scores were added to obtain the OHI-S value. The periodontal condition was assessed using the Community Periodontal Index of Treatment Needs (CPITN) and residual periodontal bone (RPB). A standard probe with a $0.5-\mathrm{mm}$ ball tip and a band between $3.5 \mathrm{~mm}$ and $5.5 \mathrm{~mm}$ was used. Gingival bleeding, calculus, and periodontal pocket depth were investigated in the dentition divided into sextants. A given sextant was examined if at least two teeth were present. 
Codes were as follows: (4) pocket $>6 \mathrm{~mm}$, (3) pocket 4 or $5 \mathrm{~mm}$, (2) calculus felt during probing, but pocket depth less than $3.5 \mathrm{~mm}$, (1) bleeding after probing, (0) healthy. The percentages of sextants with a given score in patients with and without BRONJ were computed and their distribution was compared statistically. Information about residual periodontal bone was obtained from orthopantomograms [20]. The quotient of the distance tooth apex - cemento-enamel junction and the distance tooth apex - most coronal bone level was calculated at the mesial and distal aspect of every tooth present and multiplied by 100 . One single result per tooth was averaged.

\section{Statistical analysis}

The null hypothesis $(\mathrm{Ho})$ stated that there was no difference in parameter of oral health between patients with and without BRONJ. Associations between recorded factors, i.e. independent categorical variables, and development of BRONJ, were evaluated by comparison of the differences of means of the given parameters between the groups of patients with and without BRONJ using Student's t-test or Mann-Whitney rank sum test. The chi-square test $\left(\chi^{2}\right)$ was used to assess the distribution of the independent variables between groups. Statistical analysis was performed using Sigma-Stat for Win- dows, version 2.0. Value of $p \leq 0.05$ was considered significant.

\section{Results}

The data are reported as mean with 95\% confidence interval $(95 \% \mathrm{Cl})$. Eighty-one patients were included in the assessment, 29 with bone necrosis. Table I presents the clinical characteristics of the study group. There was no difference in the mean age of patients with versus without BRONJ. Gender and BP distribution across groups was also statistically equal. The frequency of BRONJ was not related to the primary diagnosis. There was a trend towards a correlation between development of BRONJ and type of the given bisphosphonate, with zoledronate alone or in combination with other BPs being more common among BRONJ patients. Patients who developed BRONJ received significantly more infusions than those without BRONJ.

Almost all (28/29) BRONJ cases underwent previous dental surgical procedures ( 22 teeth extractions, 2 incisions of dental abscesses, 2 apicotomies, 1 implant insertion, 1 cystectomy, 1 not identified). In the control group only 7 of 52 patients underwent dental surgery during BP therapy (7 teeth extractions). A $\chi^{2}$-test showed a significant difference in the distribution of dental surgery between groups $(p=0.001)$.

Table I. Clinical characteristics of the study group

\begin{tabular}{|c|c|c|c|c|c|}
\hline Parameter & & $\begin{array}{l}\text { Patients with } \\
\text { BRONJ } \\
(n=29)\end{array}$ & $\begin{array}{c}\text { Patients } \\
\text { without BRONJ } \\
\quad(n=52)\end{array}$ & Value of $p$ & Statistics \\
\hline \multirow[t]{2}{*}{ Gender } & Male & 14 & 22 & 0.776 & $\chi^{2}$-test \\
\hline & Female & 15 & 30 & & \\
\hline \multirow[t]{2}{*}{ Age [years] } & Mean & 69.21 & 64.66 & 0.131 & Mann-Whitney Rank Sum test \\
\hline & $95 \%$ C Index & $66.06 ; 72.36$ & $61.5,67.82$ & & \\
\hline \multirow[t]{6}{*}{ Diagnosis } & MM & 11 & 16 & 0.271 & $\chi^{2}$-test \\
\hline & $\mathrm{BC}$ & 9 & 24 & & \\
\hline & PC & 6 & 5 & & \\
\hline & $\mathrm{RC}$ & 3 & 3 & & \\
\hline & $\mathrm{BrC}$ & & 3 & & \\
\hline & CC & & 2 & & \\
\hline \multirow[t]{7}{*}{ Type of BP } & Z & 17 & 24 & 0.088 & $\chi^{2}$-test \\
\hline & $\mathrm{P}$ & 8 & 7 & & \\
\hline & 1 & 1 & 14 & & \\
\hline & $Z+P$ & 2 & 1 & & \\
\hline & $Z+1$ & 1 & 4 & & \\
\hline & $P+1$ & & 1 & & \\
\hline & $Z+P+1$ & & 1 & & \\
\hline \multirow[t]{2}{*}{ No. of doses } & Mean & 33.69 & 19.8 & 0.001 & Mann-Whitney rank sum test \\
\hline & $95 \%$ C Index & $24.42,42.96$ & $14.07,25.52$ & & \\
\hline
\end{tabular}

$M M$ - multiple myeloma, $B C$ - breast carcinoma, $P C$ - prostate carcinoma, $R C$ - renal cell carcinoma, $B r C$ - bronchial carcinoma, $C C$ - colon carcinoma, $Z$-zolendronate, $P$ - pamidronate, $I$ - ibandronate 
Table II. Comparison of OHI-S, DMFT, CPITN, and RPB indices between groups

\begin{tabular}{|lccccc|}
\hline Variables & \multicolumn{2}{c}{$\begin{array}{c}\text { Patients } \\
\text { with } \\
\text { BRONJ } \\
(n=29)\end{array}$} & $\begin{array}{c}\text { Patients } \\
\text { without } \\
\text { BRONJ } \\
(n=52)\end{array}$ & $\begin{array}{c}\text { Value } \\
\text { of } p\end{array}$ & Statistics \\
\hline OHI(S) & Mean & 1.94 & 1.32 & 0.065 & t-test \\
\cline { 2 - 7 } & $95 \% \mathrm{Cl}$ & $1.18,2.7$ & $1.05,1.59$ & & \\
\hline DMFT & Mean & 26.85 & 22.87 & 0.05 & Mann-Whitney rank sum test \\
\cline { 2 - 7 } & $95 \% \mathrm{Cl}$ & $23.89,29.8$ & $21.14,24.6$ & & \\
\hline CPITN [\% of sextants] & 0 & 21.05 & 42.51 & 0.113 & $\chi^{2}$-test \\
\cline { 2 - 7 } & 1 & 13.16 & 7.29. & 0.420 & $\chi^{2}$-test \\
\cline { 2 - 7 } & 2 & 0 & 15.38 & 0.033 & $\chi^{2}$-test \\
\cline { 2 - 7 } & 3 & 65.79 & 28.34 & 0.036 & $\chi^{2}$-test \\
\hline Residual periodontal bone [\%] & Mean & 73.1 & 80.51 & 0.001 & Mann-Whitney rank sum test \\
\cline { 2 - 7 } & $95 \% \mathrm{Cl}$ & $69.8,76.38$ & $78.11,82.92$ & & \\
\hline
\end{tabular}

Table II reports the results of OHI-S, DMFT, CPITN, and RPB indices. Estimation of difference of means of OHI-S index showed an association of BRONJ and worse dental hygiene. The coincidence of BRONJ and caries computed with the DMFT index was statistically significant $(p=0.05)$. The periodontal status of patients with BRONJ was worse than those without BRONJ. The percentage of patients with less advanced periodontal disease (CPITN scores $0,1,2$ ) was greater in the control group, although this did not reach statistical significance ( $p=0.074 ; \chi^{2}$ test). The percentage of patients with deep teeth pockets (scores 3 and 4) was significantly higher among BRONJ patients ( $p=0.036 ; \chi^{2}$ test), accounting for $65.79 \%$ of this group versus only $34.82 \%$ in the control group. The level of residual periodontal bone was significantly higher in patients not having BRONJ.

\section{Discussion}

Cooperation between the Department of Hematology and Oncology and the Department of Maxillofacial Surgery in our institution was established to maintain the oral health of oncologic patients before and during chemotherapy [16]. The data recorded during this treatment protocol were used in this study to assess the relationship between oral health and development of BRONJ. Statistical analysis confirmed that the evaluated groups were comparable in terms of age, gender, diagnosis, and type of administered BPs.

In the present study the percentage of patients with deep teeth pockets was significantly higher among BRONJ cases. The main advantage of the CPITN is its ease of use. The limitation is that it does not register some signs of periodontal disease such as dental mobility and attachment loss, so pocket depth and bleeding were the indices of more advanced periodontal disease among BRONJ patients [22]. The DMFT index showed poorer dental status of BRONJ patients. The DMFT index reflects how many teeth are influenced by caries. It could be influenced by the increased number of removed teeth in BRONJ patients only if the majority of teeth were removed because of reasons other than caries. This was not the case in the present study. Therefore, the total DMFT score remained unchanged even when shifted from decayed or filled to missing. The critical $p$-value of the differences of means of OHI-S did not reach statistical significance, but it could be assumed that there is a tendency to worse dental hygiene among BRONJ patients. Larger studies on dental and periodontal conditions of patients treated with BPs might identify differences that did not reach statistical significance in this study possibly due to the small sample size. Such interpretation of the present results is consistent with clinical experience, and recently was also supported by experimental data $[23,24]$. In the series of cases reported by Marx et al., it was pointed out that the most common dental comorbidity identified in $84 \%$ of BRONJ patients was bacterial plaque, leading to the development of clinically and radiologically apparent periodontitis. Dental caries in the area of exposed bone was noted in $28.6 \%$ of cases and about $13 \%$ of patients had dental abscess formation [8]. The only other study similar to this was conducted by Carmagnola et al., in which 39 patients were enrolled during 1 year of observation. The authors attempted to prove that patients with BRONJ had poorer dental and periodontal history than non-BRONJ patients. The average DMFT index of healthy patients was 18.78 , lower than the value for patients with BRONJ (22.35); however, this 
difference was far from statistically significant ( $p=0.156)$. Residual periodontal bone support was $65.08 \%$ and $68.93 \%$ for BRONJ and nonBRONJ patients, respectively, and this difference was not significant $(p=0.118)$. Clinical evaluation of the periodontium was not performed [14]. The discrepancy with our results could be related to the small study size and supports the need for large epidemiological studies on this topic. In another study, the oral health status of patients treated with oral BPs for osteoporosis was compared to an age - and gender-matched control group without BP administration and to community data. The oral health of BP patients was poorer than controls and much worse than average for the population. DMFT scores were 29, 24, and 19 for patients on oral BPs, controls, and the community, respectively. Advanced periodontal disease was present in over $95 \%$ of patients in the first and the second group, but only $23 \%$ of the community population [25]. Another study found visible plaque and calculus in $87.5 \%$ of patients with prostate cancer and BRONJ vs. $51.4 \%$ plaque and $68.6 \%$ calculus in patients with BP administration but no signs of bone necrosis [26].

It is common in the literature that analysis of oral health associated risk factors is reduced to the causative role of dental surgical procedures in the genesis of BRONJ $[4,11,12]$. Consistent with this, dental surgery preceded the development of BRONJ in 28 of 29 patients in the present study. The fact that 7 of 52 patients from the control group underwent dental extraktions that did not lead to the development of BRONJ remains unclear, but it might be related to the duration of BPS treatment. It is also not clear whether BPs could be solely responsible for bone necrosis in patients who are simultaneously under the influence of other potentially osteonecrotic or inflammatory co-morbidities. Although long-term therapy with BPs is substantially linked to BRONJ, chemotherapy, exogenous steroids, dental surgery, and superinfection with Actinomyces are also recognized potential co-factors [27-29]. Other nonspecific risks could include vascular disorders, diabetes, smoking, or malnutrition [30]. An etiologic role of infection is interesting, based on the theory of BRONJ arising from bacterial colonization of the denuded bony surface after tooth extraction, thus creating favorable conditions for the development of chronic and therapy-resistant infection [31-33]. On the other hand, there are many reports indicating that BPs may prevent alveolar bone loss and possibly stimulate new bone formation, and might play a role in improving bone condition [19, 34]. In a study by Rocha et al., the effect of 6 months of oral BP treatment in 40 postmenopausal women with established periodontal disease was evalu- ated in a controlled, double-masked, prospective study. The BP group experienced a significant reduction in probing pocket depth, a significant increase in alveolar bone height, and a significant decrease in tooth mobility compared to the placebo group [35]. A protective action of BPs on resorption of the alveolar bone following tooth extraction was also reported in experimental animal studies [20, 23].

These facts show that interpretation of the true influence of BPs on bone health must be done extremely cautiously. In this study, patients on BPs with BRONJ had poorer oral hygiene, showed more complications of caries, and had worse periodontal status when compared with those without BRONJ. However, it is not possible to ascribe an accompanying role to BPs concerning these features. Ninety percent of patients treated with BPs for osteoporosis were also significantly medically compromised, which could influence the oral hygiene level [25]. Further, the negative influence of hospitalization on oral health was already reported [17]. These are important observations given that the majority of patients treated with intravenous BPs have significant oncologic disease, difficulties with maintenance of routine dental hygiene, and undergo repeated hospitalization. These patients are also more susceptible to oral infections due to general disability, systemic or local alterations in the host response, or multifactorial causes. Because of this, the formation of dental plaque and calculus within the limits of the mucogingival junction can lead to degradation of the periodontal attachment apparatus and consequently to development of periodontitis and subsequent involvement of alveolar bone. The incidence of jaw osteonecrosis in patients with cancer is reportedly up to fourfold higher than that in the healthy population [36, 37]. Furthermore, empiric improvement of dental hygiene was shown to reduce the incidence of BRONJ in patients with multiple myeloma and metastatic cancer [38, 39].

In conclusion, the present study is the first to demonstrate a relationship between poorer oral hygiene and the development of bone necrosis in patients on BPs. Furthermore, in almost all cases advanced dental caries or periodontal disease required surgical intervention, most commonly tooth extraction, which directly contributed to the development of BRONJ. The BPs are used successfully in the supportive treatment of cancer metastatic to bone. Therefore, much effort is required to reduce the risk of BRONJ and improve the management of this complication, which is difficult to treat. Patients receiving BPs should be offered the full range of preventive care to reduce the risk of dental complications and periodontal disease, while oncologists and dentists should be 
aware of the pathology and management of this disease.

\section{Acknowledgments}

The author is grateful to Prof. Dr. M. Griesshammer, Head of the Department of Hematology and Oncology, and PD Dr. W. Engelke, Head of the Department of Maxillofacial Surgery, Klinikum Minden, for their substantial support and establishment of cooperation between both units, which was essential for this study.

\section{References}

1. Graham R, Russell G. Bisphosphonates: mode of action and pharmacology. Pediatrics 2007; 119: 150-62.

2. Gutta R, Louis PJ. Bisphosphonates and osteonecrosis of the jaws: science and rationale. Oral Surg Oral Med Oral Pathol Oral Radiol Endod 2007; 104: 186-93.

3. Kos M, Luczak K, Godzinski J, Klempous J. Treatment of monostotic fibrous dysplasia with pamidronate. J Craniomaxillofac Surg 2004; 32: 10-5.

4. Hoff AO, Toth BB, Altundag K, et al. Frequency and risk factors associated with osteonecrosis of the jaw in cancer patients treated with intravenous bisphosphonates. J Bone Miner Res 2008; 23: 826-36.

5. Wu S, Dahut WL, Gulley JL. The use of bisphosphonates in cancer patients. Acta Oncol 2007; 46: 581-91.

6. Marx RE. Pamidronate (Aredia) and zoledronate (Zometa) induced avascular necrosis of the jaws: a growing epidemic. J Oral Maxillofac Surg 2003; 61: 1115-7.

7. Migliorati CA, Schubert MM, Peterson DE, Seneda LM. Bisphosphonate-associated osteonecrosis of mandibular and maxillary bone. An emerging oral complication of supportive cancer therapy. Cancer 2005; 104: 83-93.

8. Marx RE, Sawatari Y, Fortin M, Broumand V. Bisphosphonate-induced exposed bone (osteonecrosis/osteopetrosis) of the jaws: risk factors, recognition, prevention and treatment. J Oral Maxillofac Surg 2005; 63: 1567-75.

9. Kos M, Kuebler JF, Luczak K, Engelke W. Bisphosphonate-related osteonecrosis of the jaws: a review of 34 cases and evaluation of risk. J Craniomaxillofac Surg 2010; 38: 255-9.

10. Xiaohui Z, Jing H, Yunfeng L, Guozhu Y, En L. Effects of ibandronate-hydroxyapatite on resorptive activity of osteoclasts. Arch Med Sci 2011; 7: 53-60.

11. Bamias A, Kastritis E, Bamia C, et al. Osteonecrosis of the jaw in cancer after treatment with bisphosphonates: incidence and risk factors. J Clin Oncol 2005; 23: 8580-7.

12. Zervas K, Verrou E, Teleioudis Z, et al. Incidence, risk factors and management of osteonecrosis of the jaw in patients with multiple myeloma: a single-centre experience in 303 patients. Br J Haematol 2006; 134: 20-3.

13. Boonyapakorn T, Schirmer I, Reichart PA, Sturm I, Massenkeil G. Bisphosphonate-induced osteonecrosis of the jaws: prospective study of 80 patients with multiple myeloma and other malignancies. Oral Oncol 2008; 44: 857-69.

14. Carmagnola D, Celestino S, Abati S. Dental and periodontal history of oncologic patients on parenteral bisphosphonates with or without osteonecrosis of the jaws: a pilot study. Oral Sur Oral Med Oral Pathol Oral Radiol Endod 2008; 106: e10-5.
15. Khafagy R, Shackley D, Samuel J, O'Flynn K, Betts C, Clarke N. Complications arising in the final year of life in men dying from advanced prostate cancer. J Palliat Med 2007; 10: 705-11.

16. Elad S, Thierer T, Bitan M, Shapira MY, Meyerowitz C. A decision analysis: the dental management of patients prior to hematology cytotoxic therapy or hematopoietic stem cell transplantation. Oral Oncol 2008; 44: 37-42.

17. Terezakis E, Needleman I, Kumar N, Moles D, Agudo E. The impact of hospitalization on oral health: a systematic review. J Clin Periodontol 2011; 38: 628-36.

18. Hornby SB, Evans GP, Hornby SL, Pataki A, Glatt M, Green JR. Long-term zoledronic acid treatment increases bone structure and mechanical strength of long bones of ovariectomized adult rats. Calcif Tissue Int 2003; 72: 519-27.

19. Little DG, Smith NC, Williams PR, et al. Zoledronic acid prevents osteopenia and increases bone strength in a rabbit model of distraction osteogenesis. J Bone Miner Res 2003; 18: 1300-7.

20. Altundal H, Güvener Ö. The effect of alendronate on resorption of the alveolar bone following tooth extraction. Int J Oral Maxillofac Surg 2004; 33: 286-93.

21. Hardt CRE, Gröndahl K, Lekholm U, Wennström JL. Outcome of implant therapy in relation to experienced loss of periodontal bone support. Clin Oral Implants Res 2002; 13: 488-94.

22. Benigeri M, Brodeur JM, Payette M, Charbonneau A, Ismail A. Community periodontal index of treatment needs and prevalence of periodontal conditions. J Clin Periodontol 2000; 27: 308-12.

23. Jee JH, Lee $W$, Lee $B D$. The influence of alendronate on the healing of extraction sockets of ovariectomized rats assessed by in vivo micro-computed tomography. Oral Surg Oral Med Oral Pathol Oral Radiol Endod 2010; 110: e47-53.

24. Aghaloo TL, Kang B, Sung EC, et al. Periodontal disease and bisphosphonates induce osteonecrosis of the jaws in the rat. J Bone Miner Res 2011; 26: 1871-82.

25. Kunchur R, Goss AN. The oral health status of patients on oral bisphosphonates on osteoporosis. Aust Dent J 2008; 53: 354-57.

26. Walter C, Al-Nawas B, Grötz KA, et al. Prevalence and risk factors of bisphosphonate-associated osteonecrosis of the jaw in prostate cancer patients with advanced disease treated with zoledronate. Eur Urol 2008; 54: 1066-72.

27. Mirzai R, Chang C, Greenspan A, Gershwin ME. The pathogenesis of osteonecrosis and the relationships to corticosteroids. J Asthma 1999; 36: 77-95.

28. Khamaisi M, Regev E, Yarom N, et al. Possible association between diabetes and bisphosphonate-related jaw osteonecrosis. J Clin Endocrinol Metab 2007; 92: 1172-5.

29. Kos M, Brusco D, Kuebler JF, Engelke W. Clinical comparison of patients with osteonecrosis of the jaws, with and without a history of bisphosphonates administration. Int J Oral Maxillofac Surg 2010; 39: 1097-102.

30. Landesberg R, Woo V, Cremers S, et al. Potential pathophysiological mechanisms in osteonecrosis of the jaw. Ann N Y Acad Sci 2011; 1218: 62-79.

31. Ganguli A, Steward C, Butler SL, et al. Bacterial adhesion to bisphosphonate coated hydroxyapatite. J Mater Sci Mater Med 2005; 16: 283-7.

32. Kobayashi Y, Hiraga T, Ueda A, et al. Zoledronic acid delays wound healing of the tooth extraction socket, inhibits oral epithelial cell migration, and promotes proliferation and adhesion to hydroxyapatite of oral bacte- 
ria, without causing osteonecrosis of the jaw, in mice. J Bone Mineral Metab 2010; 28: 165-75.

33. Kos M. Bisphosphonates promote jaw osteonecrosis through facilitating bacterial colonisation. Med Hypotheses 2011; 77: 214-5.

34. Pampu AA, Dolanmaz D, Tüz HH, Karabacakoglu A. Experimental evaluation of the effects of zoledronic acid on regenerate bone formation and osteoporosis in mandibular distraction osteogenesis. J Oral Maxillofac Surg 2006; 64: 1232-6.

35. Rocha ML, Malacara JM, Sanchez-Marin FJ, Vazquez de la Torre CJ, Fajardo ME. Effect of alendronate on periodontal disease in postmenopausal women. A random ized placebo-controlled trial. J Periodontol 2004; 75: 1579-85.

36. Tarassoff P, Csermak K. Avascular necrosis of the jaws: risk factors in metastatic cancer patients. J Oral Maxillofac Surg 2003; 61: 1238-9.

37. Schwartz HC. Osteonecrosis and bisphosphonates: correlation versus causation. J Oral Maxillofac Surg 2004, 62: 763-4.

38. Dimopoulos MA, Kastritis E, Bamia C, et al. Reduction of osteonecrosis of the jaw (ONJ) after implementation of preventive measures in patients with multiple myeloma treated with zoledronic acid. Ann Oncol 2009; 20: 117-20.

39. Ripamonti Cl, Maniezzo M, Campa T, et al. Decreased occurrence of osteonecrosis of the jaw after implementation of dental preventive measures in solid tumour patients with bone metastases treated with bisphosphonates. The experience of the National Cancer Institute of Milan. Ann Oncol 2009; 20: 137-45. 